\title{
SEASONALITY AND ASSOCIATION OF METEOROLOGICAL FACTORS WITH RESPIRATORY VIRUSES DETECTION IN NON-HOSPITALIZED CHILDREN WITH COMMUNITY-ACQUIRED PNEUMONIA IN A TROPICAL REGION
}

Amanda C. Nascimento-Carvalho ${ }^{a}$, Igor C. Borges ${ }^{b}$, Ana-Luisa Vilas-Boas ${ }^{c}$, Maria-Socorro H. Fontourac ${ }^{c}$, Tytti Vuorinen ${ }^{d}$, Olli Ruuskanene, Cristiana M. Nascimento-Carvalhoc the PNEUMOPAC-Efficacy Study Groupc

Affiliations:

aBahiana School of Medicine, Bahiana Foundation for Science Development, Salvador, Brazil

Federal University of Bahia School of Medicine, Salvador, Bahia, Brazil

Funding:

E-mail: carvalhoacn@hotmail.com

'Department of Pediatrics, Federal University of Bahia School of Medicine, Salvador, Brazil

dDepartment of Clinical Virology, Turku University Hospital, and Department of Virology, Turku University, Turku, Finland

eDepartment of Pediatrics, University of Turku, Turku, Finland

This work was supported by a grant from the Bahia State Agency for Research Funding (FAPESB) (Grant No. PPSUS №. SUS0023/2013). CMN-C is a senior investigator at the Brazilian Council for Science and Technological Development (CNPq).

\section{BACKGROUND}

Community-acquired pneumonia (CAP) is the leading cause of death and hospitalization among children under-5 years worldwide. Information on the seasonal distribution and association with meteorological factors of respiratory viruses, particularly in tropical regions, is scarce. We aimed to investigate the seasonal distribution and the association of meteorological factors with the frequency of detection of respiratory viruses in children with CAP in a tropical region.

\section{METHODS}

In a 54-month period, non-hospitalized patients, aged 2-59 months, were diagnosed with CAP in Salvador, Northeast Brazil, had nasopharyngeal aspirate samples collected and tested for 16 respiratory viruses by PCR (Anyplex [TM] II RV 16. Seegene, Seoul, South Korea). Data about rainfall, relative humidity, air temperature, and sunshine were collected. Time series analysis using Prais-Winsten generalized linear regression was used for identification of seasonality and association with meteorological factors.

\section{RESULTS}

Out of 774 cases, $708(91 \%)$ had $\geq 1$ respiratory virus found. The monthly number of rhinovirus, adenovirus, enterovirus, respiratory syncytial viruses $A(R S V A)$ and $B(R S V B)$, influenza viruses $A($ Flu $A$ ) and $B$ (Flu $B$ ) detections showed seasonal distribution. Rainfall was associated with the number of detections of rhinovirus, adenovirus, RSVB, Flu A, and coronavirus 229E. Relative humidity was associated with the number of detections of rhinovirus, adenovirus, RSVA, RSVB, parainfluenza 2, human metapneumovius (hMPV), Flu A, and coronavirus NL63. Air temperature was associated with the number of detections of hMPV and Flu A. Hours of sunshine was associated with the number of detections of hMPV.

\section{CONCLUSION}

Seasonality and meteorological factors probably play a role in the frequency of detection of the majority of the investigated viruses making possible planning of vaccine prevention regarding the season of the year in tropical regions. 